\title{
Effects of Dietary Fish Oil on Intestinal Adaptation in 20-Day-Old Weanling Rats After Massive Ileocecal Resection
}

\author{
QING YANG AND NANCY D. KOCK \\ Departments of Pediatrics [Q.Y.] and Pathology/Comparative Medicine [N.D.K.], Wake Forest University Health Science, Winston-Salem, \\ North Carolina 27157
}

\begin{abstract}
Long chain polyunsaturated fatty acids (LCPUFA) seem to be the most trophic macronutrients in inducing intestinal adaptation in adult short bowel syndrome (SBS), although their effects on intestinal adaptation in infants with SBS remain unknown. It is hypothesized that a high fat diet enriched with n-3 LCPUFA derived from fish oil (FO) will increase intestinal adaptation compared with a diet dominated by n-6 PUFA from corn oil (CO) in weanling SBS rats after massive ileocecal resection (ICR). Twentyday-old rats were sorted into four groups, CO-sham, FO-sham, CO-ICR, and FO-ICR groups, and fed $a d$ lib with the CO or FO diet, respectively, for $7 \mathrm{~d}$ after sham or ICR surgery. Compared with CO-ICR rats, FO-ICR rats consumed less diet per gram of weight gain, had less diarrhea and fecal fat excretion, and demonstrated a tendency toward better weight gain. The mucosal mass, DNA and RNA levels of the colon and RNA levels of the distal jejunum, and the colonic mucosal area (\%) were significantly higher in FO-ICR rats than in CO-ICR rats. These results suggest that the beneficial effect of dietary FO is associated with better adaptation in the colon in weanling rats after ICR. (Pediatr Res 68: 183-187, 2010)
\end{abstract}

$\mathrm{I}^{\mathrm{n}}$ ntestinal adaptation in short bowel syndrome (SBS) is a compensatory physiologic response that occurs after loss of mucosal surface area and is important in restoring the digestive and absorptive capacity of the intestine. Enteral nutrition plays a key role in the adaptive process in SBS, which depends on both the presence (1) and quality (2) of enteral nutrients. Enteral fats seem to be the most trophic macronutrients in inducing adaptation (3-5). High fat diets (5-8) and diets enriched with long chain fatty acids $(9,10)$ have been shown to be more effective, and especially, diets rich in long chain polyunsaturated fatty acids (LCPUFA) are the most beneficial in stimulating mucosal adaptation $(4,11)$. Fish oil (FO) is a main source of n-3 LCPUFA eicosapentaenoic acid (EPA) and docosahexaenoic acid (DHA). Rats with SBS were fed with a diet containing $15 \%$ FO had better mucosal adaptation than those fed with a diet containing less highly unsaturated fats (11). However, the impact of a diet with combination of high fat and LCPUFA on mucosal adaptation is unclear. Moreover,

Correspondence: Qing Yang, M.D., Ph.D., Division of Neonatology, Department of Pediatrics, Wake Forest University Health Science, Medical Center Blvd., WinstonSalem, NC 27157; e-mail: qyang@wfubmc.edu

Supported by the developmental fund of Department of Pediatrics in Wake Forest University Health Science, Winston-Salem. those studies (4-11) used adult SBS rats, and thus, the effect of dietary fat on intestinal adaptation in infantile SBS remains unknown.

Recently, we developed an SBS model in 20-d-old weanling rats with $50 \%$ ileocecal resection (ICR) and partial colon resection (12). This model is more appropriate for studying human infantile SBS, which most often occurs after surgical treatment for necrotizing enterocolitis in premature infants $(13,14)$, as the intestinal resection is similar in both. Using this model in this study, we compared the trophic effects of a high fat diet enriched with n-3 LCPUFA from FO to one containing the same amount of fat, but with n-6 PUFA from corn oil (CO), on the adaptive responses in the residual jejunum and colon.

\section{METHODS}

Animals, diets, and surgical procedures. The experimental protocol for this study was approved by the Institutional Animal Care and Use Committee at Wake Forest University Health Sciences. Forty-six male weanling SpragueDawley rats were separated from their dams at $20 \mathrm{~d}$ of age (weight range 42-60 g). Eighteen of these underwent sham surgery and afterward nine were fed with the CO diet (CO-sham) and nine were fed with the FO diet (FO-sham). The remaining 28 rats underwent ICR and afterward half were fed with the CO diet (CO-ICR) and half were fed with the FO diet (FO-ICR). The rats were allowed access only to water for 4-6 h before surgery and then provided with the liquid diet for $7 \mathrm{~d}$ after surgery. Water was available only for the first $24 \mathrm{~h}$ after surgery when intake of the liquid diet was minimal.

The liquid diet, Lieber-DeCarli regular control rat diet (Dyets no. 710027; Dyets Inc., Bethlehem, PA), was used as a base diet. The high fat diets were made using $11.4 \mathrm{~mL}$ of FO (Menhaden oil, Dyets no. 402940,) or CO and were mixed thoroughly with $221.78 \mathrm{~g}$ of the base diet before adding water up to $1 \mathrm{~L}$. This mixture was then homogenized for $30 \mathrm{~s}$ using a Polytron-like mixer according to the instructions of the manufacturer. The FO diet contained $20 \%$ FO and $17.2 \% \mathrm{CO}$ (wt/wt fat), whereas the CO diet had $37.2 \%$ CO. Both diets contained $41 \%$ of the energy as fat (Table 1) compared with $14 \%$ in regular rat chow (ProLab RMH 3000, LabDiet). Dietary lipids were extracted (15), and the dietary fatty acid compositions were analyzed (16) (Table 2). In all cases, the diets were used within $48 \mathrm{~h}$ after preparation.

The ICR surgery was performed as previously described (12). Briefly, surgery was performed aseptically, using inhaled $2 \%$ isoflurane and oxygen for anesthesia throughout the procedure. The length of small bowel (jejunum + ileum) was measured by placing a suture thread along the antimesenteric border of the intestine from the ligament of Treitz to ileum-

Abbreviations: CO, corn oil; DHA, docosaheaenoic acid; EPA, eicosapentaenoic acid; FO, fish oil; ICR, ileocecal resection; LCPUFA, long chain polyunsaturated fatty acid; PUFA, polyunsaturated fatty acid; PYY, peptide YY; SBS, short bowel syndrome 
Table 1. Nutritional profiles of $\mathrm{CO}$ and $\mathrm{FO}$ diets

\begin{tabular}{lcc}
\hline & CO diet $[\mathrm{g} / \mathrm{L}(\% \mathrm{kcal})]$ & FO diet $[\mathrm{g} / \mathrm{L}(\% \mathrm{kcal})]$ \\
\hline Protein & $45.0(16.6)$ & $45.0(16.6)$ \\
Carbohydrate & $115.2(42.4)$ & $115.2(42.4)$ \\
Fat & $49.5(41.0)$ & $49.5(41.0)$ \\
$\quad$ Corn oil & $18.4(15.2)$ & $8.5(7.0)$ \\
(\% wt/wt) & $(37.2)$ & $(17.2)$ \\
Olive oil & $28.4(23.5)$ & $28.4(23.5)$ \\
(\% wt/wt) & $(57.4)$ & $(57.4)$ \\
Salflower oil & $2.7(2.2)$ & $2.7(2.2)$ \\
(\% wt/wt) & $(5.5)$ & $(5.5)$ \\
Fish oil $*$ & - & $(20.0)$ \\
$(\% \mathrm{wt} / \mathrm{wt})$ & & 8.8 \\
Mineral mix $\dagger$ & 8.8 & 2.5 \\
Vitamin mix $\ddagger$ & 2.5 & 1.1 \\
kcal/mL & 1.1 &
\end{tabular}

* Dyets no. 402940, Menhaden oil.

$\dagger$ Dyets no. 210011.

\$Dyets no. 310011 .

Table 2. Fatty acid compositions of $\mathrm{CO}$ and $\mathrm{FO}$ diets

\begin{tabular}{ccc}
\hline & CO diet & FO diet \\
\hline Fatty acid (\%) & & \\
14:0 & 0.8 & 1.8 \\
$16: 0$ & 11.8 & 13.5 \\
$16: 1$ & 0.5 & 2.7 \\
$18: 0$ & 2.6 & 3.0 \\
$18: 1 \mathrm{n} 9$ & 51.6 & 49.2 \\
$18: 1 \mathrm{n} 7$ & 1.5 & 2.2 \\
$18: 2 \mathrm{n} 6$ & 30.3 & 20.3 \\
$18: 3 \mathrm{n} 3$ & 1.1 & 1.2 \\
$18: 4 \mathrm{n} 3$ & - & 0.5 \\
20:4 n6 & - & 0.4 \\
20:5 n3 & - & 2.3 \\
22:5 n3 & - & 0.5 \\
22:6 n3 & - & 1.8 \\
n-6/n-3 PUFA ratio & 27.1 & 3.3 \\
\hline
\end{tabular}

cecum junction. In rats undergoing ICR, the segment of intestine between the fifth and sixth mesenteric vessel arcade proximal to the ileocecal junction and $1 \mathrm{~cm}$ distal to the cecum in the ascending colon was removed after ligation of the mesenteric vessels, resulting in the removal of an average of $29 \mathrm{~cm}$ of small intestine, which was $\sim 50 \%$ of the small bowel. End-to-end anastomosis of the jejunum and proximal colon was performed using interrupted 7-0 Vicryl ophthalmic suture. The sham surgery rats were subjected to bowel transection at the same proximal location as the ICR group, with subsequent anastomosis at the same site. After surgery, the rats were hydrated with $1 \mathrm{~mL}$ of warm saline by i.p. instillation, and the abdomen was closed. The rats were held in an infant incubator at $35^{\circ} \mathrm{C}$ for at least $30 \mathrm{~min}$ after surgery, until fully recovered from anesthesia, and then returned to conventional individual housing cages.

Data and sample collection. Body weights, food intake, and stool changes were recorded daily. At the end of the seventh postoperative day, the rats were killed humanely by cervical dislocation, and the intestinal tract from the gastric pylorus to the rectum was removed. After collecting stool samples from the colon, $1 \mathrm{~cm}$ segments of intestine on either sides of the anastomosis sites were discarded, and the adjacent $1 \mathrm{~cm}$ of jejunum (both ICR and sham groups) and $1 \mathrm{~cm}$ of proximal colon (ICR group), as well as $1 \mathrm{~cm}$ section of colon directly adjacent to the cecum (sham group), were opened longitudinally and preserved in $10 \%$ buffered formalin for at least $24 \mathrm{~h}$. The sections were trimmed, embedded in paraffin, processed routinely for histology, cut at $6 \mu \mathrm{m}$, and stained with hematoxylin and eosin (H\&E). The remaining intestine was thoroughly rinsed with ice-cold saline and gently blotted, and the weights and lengths of the small bowel and colon were recorded. Five centimeters long segments of the remaining distal jejunum, middle jejunum between the ligament of Treitz and anastomosis site, and colon distal to the anastomosis site in the ICR rats as well as corresponding segments in the sham rats were collected. The segments were opened longitudinally, and the mucosa was gently scraped from the underlying intestinal wall using a glass slide, weighed, frozen in liquid nitrogen, and stored in $-80^{\circ} \mathrm{C}$. The stool samples were stored at $-20^{\circ} \mathrm{C}$.

Total fecal fat. One gram of wet stool in a weighed glass tube was dried at $60^{\circ} \mathrm{C}$ under $\mathrm{N}_{2}$ flow. The stool water content was defined as the difference between wet and dry stool. Total fecal fat was extracted from the dry stool using methanol and chloroform as previously described (15) with modifications. The dry stool was ground as fine as possible, and $2 \mathrm{~mL}$ of methanol and chloroform were added followed by $1 \mathrm{~mL}$ of normal saline. After vigorously shaking, the chloroform phase containing the lipid extracted was carefully transferred into a new weighed glass tube and dried at $60^{\circ} \mathrm{C}$ under $\mathrm{N}_{2}$ flow. The glass tube was weighed again after the lipid extraction was dried, and the total fecal fat was expressed as micrograms lipid per gram dry stool.

Mucosal DNA and RNA extraction, and protein and maltase assays. Mucosal DNA and RNA were extracted from frozen tissue using DNAzol and TRIzol reagents (Invitrogen, Carlsbad, CA), respectively, as suggested by the manufacturer, and expressed as micrograms per centimeter of bowel. Mucosal proteins were determined using a DC Protein Assay Kit (Bio-Rad, Hercules, CA) according to the manufacturer's protocol and expressed as milligrams per centimeter of bowel. Intestinal disaccharidase (maltase) activity was assayed using previously described method (17). Glucose production was quantified using a Glucose Oxidase Reagent (Pointe Scientific, Inc., Canton, MI), and maltase activity was expressed as micromoles of glucose per gram of protein per minute.

Histology and morphometry. Histologic examination of the distal jejunum and proximal colon was done by a board certified veterinary pathologist. Length and width of small intestine villi, colonic mucosal thickness, and mitotic indices were determined qualitatively, and other lesions were noted. Morphometric measures of colonic mucosal area were determined using NIH Image 1.62. Values were obtained by tracing full thickness, $3 \mathrm{~mm}$ sections of the colon, followed by tracing only the underlying submucosa, which was subsequently subtracted from the whole. The magnification was set at $4 \times$. Mucosal values were reported as percentages of the entire sections.

Statistical analyses. All results are expressed as mean \pm SEM. Mean values between groups were compared using a one-way ANOVA followed by Bonferroni posttests. The daily weight of each group was compared using a two-way (age and group) ANOVA for repeated measures followed by Bonferroni posttests. A $p<0.05$ was considered statistically significant.

\section{RESULTS}

Weight and food intake. Figure 1 shows the daily percentage of preoperative body weight in the sham and ICR groups during the 7-d-feeding period after surgery. Preoperative body weights were similar among the four groups $(49.33 \pm 2.11 \mathrm{~g}$ in the CO-sham, $50.34 \pm 2.44 \mathrm{~g}$ in the FO-sham, $50.42 \pm$ $1.54 \mathrm{~g}$ in the CO-ICR, and $49.98 \pm 1.49$ in the FO-ICR group). Postoperative body weights declined $\sim 10 \%$ in the sham groups and $15 \%$ in the ICR groups $1 \mathrm{~d}$ after surgery. The

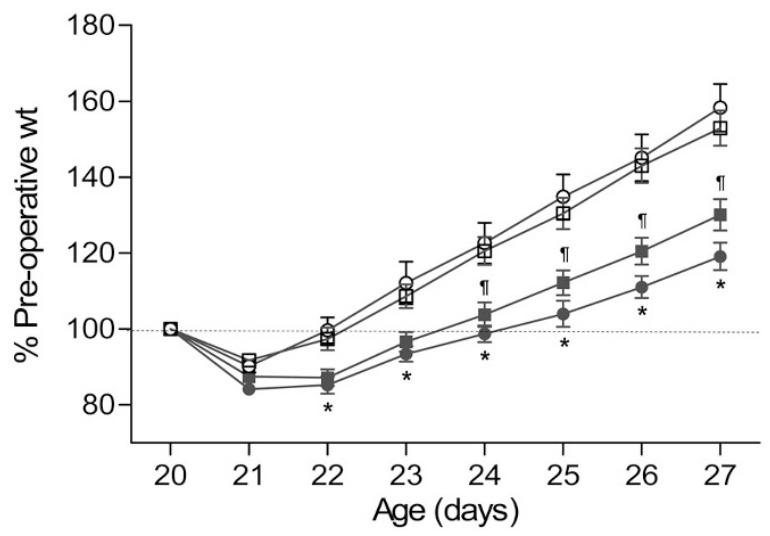

Figure 1. Daily body weight. Surgery was performed at the age of $20 \mathrm{~d}$. The daily postoperative body weight is expressed as $\%$ of preoperative weight. $\bigcirc$ CO-sham $(n=9)$, $\bullet$ CO-ICR $(n=14), \square$ FO-sham $(n=9)$, and $\square$ FO-ICR $(n=14)$ rats. ${ }^{*} p<0.05$, CO-ICR $v s$. CO-sham rats; and $\mathbb{I} p<0.05$, FO-ICR vs. FO-Sham rats at the same day. 
rats took only $1-5 \mathrm{~mL}$ liquid diets during the first $24 \mathrm{~h}$ after surgery. Thus, dehydration mainly contributed to the $10 \%$ weight loss in the first postoperative day, although the $5 \%$ additional weight loss in the ICR groups was attributed to the weight of the segment of bowel surgically removed. The bowel segments removed from the two ICR groups were similar in length $(29.0 \pm 0.9 \mathrm{~cm}$ in the CO-ICR and $28.6 \pm$ $1.00 \mathrm{~cm}$ in the FO-ICR group). By the second and fourth postoperative day, the body weights of the rats in the sham and ICR groups, respectively, were back to baseline. Both sham groups displayed similar growth rates. Both ICR groups weighed significantly less than their sham counter groups, from the second postoperative day in the CO-ICR group and from the fourth postoperative day in the FO-ICR group throughout the remaining experimental period $(p<0.05)$. FO-ICR rats tended to have better weight gains than CO-ICR rats during the last three postoperative days, although this was

Table 3. Intestinal weight and length in sham and ICR rats fed with $\mathrm{CO}$ or $\mathrm{FO}$ diets

\begin{tabular}{lcccc}
\hline & $\begin{array}{c}\text { CO-sham } \\
(\mathrm{n}=9)\end{array}$ & $\begin{array}{c}\text { FO-sham } \\
(\mathrm{n}=9)\end{array}$ & $\begin{array}{c}\text { CO-ICR } \\
(\mathrm{n}=14)\end{array}$ & $\begin{array}{c}\text { FO-ICR } \\
(\mathrm{n}=14)\end{array}$ \\
\hline Small intestine & & & & \\
$\quad$ Weight $(\mathrm{g})$ & $4.1 \pm 0.2$ & $4.0 \pm 0.2$ & $3.2 \pm 0.1^{*}$ & $3.4 \pm 0.1 \dagger$ \\
$\quad$ Length (cm) & $80.8 \pm 1.8$ & $81.8 \pm 2.5$ & $45.6 \pm 1.3^{*}$ & $49.5 \pm 1.7 \dagger$ \\
$\quad$ mg/cm & $50.6 \pm 1.0$ & $48.8 \pm 1.6$ & $71.1 \pm 2.3^{*}$ & $68.7 \pm 2.3 \dagger$ \\
Colon & & & & \\
$\quad$ Weight (g) & $0.5 \pm 0.01$ & $0.6 \pm 0.03$ & $0.7 \pm 0.04^{*}$ & $0.7 \pm 0.04$ \\
$\quad$ Length (cm) & $11.0 \pm 0.2$ & $11.2 \pm 0.4$ & $10.5 \pm 0.4$ & $10.3 \pm 0.4$ \\
mg/cm & $45.4 \pm 1.1$ & $49.3 \pm 1.3$ & $61.9 \pm 1.6^{*}$ & $64.5 \pm 0.9 \dagger$ \\
\hline
\end{tabular}

$*$ Values are mean \pm SEM different from CO-sham, $p<0.05$.

$\dagger$ Values are mean \pm SEM different from FO-sham, $p<0.05$. not statistically significant. The weights at the seventh postoperative day in each group were $79.84 \pm 1.78 \mathrm{~g}$ in the CO-sham, $78.85 \pm 3.02 \mathrm{~g}$ in the FO-sham, $59.69 \pm 1.81 \mathrm{~g}$ in the CO-ICR, and $64.68 \pm 2.11 \mathrm{~g}$ in the FO-ICR.

The average diet intake (gram of diet/gram of weight gain/d) from the fifth to the seventh postoperative days was not different between the CO-sham $(7.63 \pm 0.36)$ and FOsham rats $(8.05 \pm 0.51)$. The $\mathrm{CO}-\mathrm{ICR}$ rats displayed hyperphagia and consumed $18.26 \pm 1.87 \mathrm{~g}$ of diet per gram of weight gain per day in the last $3 \mathrm{~d}$, markedly more than that of the CO-sham and FO-ICR $(11.50 \pm 0.79)$ rats $(p<0.05)$. The food intake of the FO-ICR rats was similar to that of the FO-sham rats.

Stool patterns and total fecal fat content. Diarrhea with a foul odor was observed in the ICR rats as early as $36 \mathrm{~h}$ after surgery and continued throughout the entire experimental period. The water content per gram of wet stool was $0.78 \pm$ $0.02 \mathrm{~g}$ in the CO-ICR group and $0.75 \pm 0.01 \mathrm{~g}$ in the FO-ICR group, both more than that of their respective sham counterparts $(0.69 \pm 0.01 \mathrm{~g}$ in the CO-sham group and $0.69 \pm 0.01 \mathrm{~g}$ in the FO-sham group; $p<0.05)$. The total fecal fat, defined as $\mathrm{mg} / \mathrm{g}$ of dry stool, was 5.3-fold higher in the CO-ICR group $(356.90 \pm 17.70)$ than in the CO-sham group $(67.18 \pm 4.60)$, and 6.7-fold higher in the FO-ICR group $(272.30 \pm 21.16)$ than in the FO-sham group (40.90 \pm 5.51$)$. However, the FO-ICR group had lower total fecal fat than the CO-ICR group ( $p<0.05)$. All sham rats had normal stool.

Intestinal adaptation. Rats in both ICR groups had significantly increased small intestinal mass $(\mathrm{mg} / \mathrm{cm})$, despite decreased total lengths and weights, compared with their respective sham groups $7 \mathrm{~d}$ after surgery $(p<0.05)$. In contrast, the

Table 4. Intestinal mucosa weights, contents of protein, DNA and RNA, maltase activities, and colon mucosal areas in sham and ICR rats fed with $\mathrm{CO}$ or $\mathrm{FO}$ diets

\begin{tabular}{|c|c|c|c|c|}
\hline & $\begin{array}{l}\text { CO-sham } \\
(\mathrm{n}=9)\end{array}$ & $\begin{array}{l}\text { FO-sham } \\
(\mathrm{n}=9)\end{array}$ & $\begin{array}{l}\text { CO-ICR } \\
(\mathrm{n}=14)\end{array}$ & $\begin{array}{l}\text { FO-ICR } \\
(\mathrm{n}=14)\end{array}$ \\
\hline \multicolumn{5}{|l|}{ Wet weight $(\mathrm{mg} / \mathrm{cm})$} \\
\hline Mid-jejunum & $10.5 \pm 0.7$ & $13.2 \pm 0.6$ & $25.1 \pm 1.2^{*}$ & $24.1 \pm 1.0 \dagger$ \\
\hline Distal jejunum & $13.0 \pm 1.0$ & $13.6 \pm 0.9$ & $24.9 \pm 1.3^{*}$ & $26.2 \pm 1.4 \dagger$ \\
\hline Colon & $11.7 \pm 0.3$ & $11.3 \pm 0.5$ & $18.9 \pm 0.8^{*}$ & $22.4 \pm 1.0 \dagger \ddagger$ \\
\hline \multicolumn{5}{|l|}{ Protein $(\mathrm{mg} / \mathrm{cm})$} \\
\hline Mid-jejunum & $1.4 \pm 0.1$ & $1.7 \pm 0.2$ & $3.0 \pm 0.2^{*}$ & $3.0 \pm 0.1 \dagger$ \\
\hline Distal jejunum & $1.8 \pm 0.1$ & $1.4 \pm 0.1$ & $3.1 \pm 0.2 *$ & $3.9 \pm 0.2 \dagger$ \\
\hline Colon & $1.4 \pm 0.2$ & $1.3 \pm 0.1$ & $2.1 \pm 0.2 *$ & $2.3 \pm 0.2 \dagger$ \\
\hline \multicolumn{5}{|l|}{ DNA $(\mu \mathrm{g} / \mathrm{cm})$} \\
\hline Mid-jejunum & $57.5 \pm 4.8$ & $56.7 \pm 5.0$ & $81.8 \pm 4.3^{*}$ & $73.7 \pm 4.0 \dagger$ \\
\hline Distal jejunum & $53.0 \pm 3.6$ & $63.1 \pm 0.1$ & $81.3 \pm 7.3^{*}$ & $88.0 \pm 6.0 \dagger$ \\
\hline Colon & $38.5 \pm 1.8$ & $37.8 \pm 6.3$ & $50.2 \pm 4.7$ & $79.3 \pm 4.5+t$ \\
\hline \multicolumn{5}{|l|}{ RNA $(\mu \mathrm{g} / \mathrm{cm})$} \\
\hline Mid-jejunum & $73.8 \pm 6.6$ & $104.2 \pm 8.0$ & $186.8 \pm 9.6^{*}$ & $207.4 \pm 14.3 \dagger$ \\
\hline Distal jejunum & $94.8 \pm 10.6$ & $109.6 \pm 13.9$ & $173.5 \pm 9.5^{*}$ & $220.7 \pm 10.7 \dagger+$ \\
\hline Colon & $83.0 \pm 4.2$ & $83.4 \pm 4.6$ & $152.2 \pm 8.8^{*}$ & $185.0 \pm 12.2 \dagger \dagger$ \\
\hline \multicolumn{5}{|c|}{$\begin{array}{l}\text { Maltase activity } \\
\quad(\mu \mathrm{mol} \text { glucose/gram protein/min) }\end{array}$} \\
\hline Mid-jejunum & $0.5 \pm 0.1$ & $0.5 \pm 0.1$ & $0.5 \pm 0.04$ & $0.5 \pm 0.03$ \\
\hline Distal jejunum & $0.5 \pm 0.03$ & $0.5 \pm 0.04$ & $0.6 \pm 0.03^{*}$ & $0.6 \pm 0.03$ \\
\hline Colon mucosal area $(\%) \S$ & $52.6 \pm 1.4$ & $55.8 \pm 1.2$ & $69.2 \pm 1.3^{*}$ & $74.4 \pm 1.2 \dagger t$ \\
\hline
\end{tabular}

$*$ Values are mean \pm SEM different from CO-sham, $p<0.05$.

$\dagger$ Values are mean \pm SEM different from FO-sham, $p<0.05$.

\$Values are mean \pm SEM different from CO-ICR, $p<0.05$.

$\S$ Morphometry data were obtained from 5 rats of each sham group and 7 rats of each ICR group. 
lengths of the colons of the ICR groups were similar to their shams, but tended to be heavier, although only significantly so in the CO-ICR rats $(p<0.05)$, resulting in substantially higher colonic mass in the ICR groups than the respective sham groups ( $p<0.05$; Table 3 ). Table 4 shows the significant increases in mucosal weights and amounts of DNA, RNA, and protein in the mid-jejunum and distal jejunum and colon $7 \mathrm{~d}$ after surgery in the ICR groups. In comparing the two ICR groups, the FO-ICR rats had significantly higher mucosal mass, DNA and RNA levels in the colon, and higher RNA levels in distal jejunum than those in the CO-ICR rats. In addition, mucosal maltase activity in the mid-jejunal segment was not different between the four groups but was significantly increased in the distal jejunum in the CO-ICR rats compared with $\mathrm{CO}$-sham rats $(p<0.05)$.

Histology. Qualitative histologic differences were noted in the small intestine. The ICR groups seemed to have thicker small intestinal villi, and more mitoses among enterocytes, compared with the sham groups. There were no differences noted in the small intestinal sections between the CO-ICR and FO-ICR groups. The changes in the colon of the ICR groups were more dramatic compared with sham groups, with obvious mucosal thickening and more abundant mitoses, often nearer the luminal surface, compared with the sham groups, whereas mitoses were confined to the base of the glands in the sham groups. Morphometric measurements of the colon showed that the FO-ICR rats had significantly more mucosal area than the CO-ICR rats $(p<0.05$; Table 4$)$.

\section{DISCUSSION}

Luminal nutrients are the most influential and have direct relevance to the role of enteral nutrition as primary therapy for SBS, because mucosal adaptation will not occur in the absence of enteral feeding (1). However, not all luminal nutrients have comparable trophic effects (2). Certain lipids, primarily PUFA (18), particularly LCPUFA $(4,11)$ are highly trophic to the small intestine in adult SBS rats. This study reports, for the first time, the effects of two different dietary fats on intestinal adaptation in infant SBS rats. In contrast to adult normal and SBS rats, the gastrointestinal tract of weanling rats is more reflective of the developing human intestine (19). In addition, after ICR, the weanling rats developed signs similar to those in humans with SBS, including diarrhea with a foul odor, hyperphagia, and poor weight gain, which makes this weanling SBS rat model (12) more appropriate for modeling human infantile SBS.

This study demonstrates the effects of different dietary fats on intestinal adaptation in weanling SBS rats. Using this model, we found that ICR rats fed with the FO diet high in n-3 LCPUFA, EPA, and DHA had less diarrhea. Hyperphagia in humans with SBS is considered a compensatory mechanism to diarrhea to improve the net nutrient absorption (20). Thus, the decreased diarrhea noted in the FO-ICR rats might explain their diminished food intake and the reduction in fecal fat excretion (steatorrhea), along with the tendency toward better weight gain compared with the ICR rats on the $\mathrm{CO}$ diet high in n-6 PUFA. This suggested that the FO diet is more effective in stimulating intestinal adaptation in weanling SBS rats, although the mechanism for this remains unclear. Vanderhoof et al. (11) found that plasma peptide YY (PYY), a potent regulator of intestinal secretion and motility (21), was increased in FO-fed SBS rats compared with other dietary oils (11) although small bowel resection alone could elevate the release of PYY $(11,22)$. It is postulated that the secretion of this gastrointestinal hormone, which slows intestine motility and lengthens transit time $(21,22)$, might be increased in response to the n-3 LCPUFA from FO, thus resulting in less diarrhea and increased mucosal contact with nutrients, allowing for increased absorption of intestinal nutrients.

High fat diets accelerate postresection intestinal adaptation in adult rats with SBS (5). To maximize the beneficial impact of enteral lipids, our two diets were formulated to increase the fat calories to $41 \%$ by adding $20 \%$ FO (wt/wt fat) or $20 \%$ CO. The major difference between the FO and $\mathrm{CO}$ diets is in the fatty acid composition. The FO diet was enriched with the n-3 LCPUFA, EPA, and DHA, contained an 8-fold lower ratio of n-6/n-3 PUFA compared with the CO diet (Table 2). The FO diet was better than the $\mathrm{CO}$ diet in promoting mucosal adaptation in ICR rats, a finding consistent with that of Vanderhoof et al. (11). However, interestingly, the significant increases in mucosal mass, DNA, and RNA in ICR rats fed with the FO diet occurred primarily in the residual proximal colon, not in the small intestine (except for RNA in the distal jejunum). In this study, histologic examination demonstrated increased colonic mucosal area in the FO-ICR rats compared with the CO-ICR rats. Although data on colonic adaptation were not available in the study of Vanderhoof et al., (11) they did report significantly increased mucosal adaptation in the residual duodenum and ileum of adult SBS rats fed with the FO diet. The discrepancy between the previous study (11) and this study may be explained by differences in mucosal adaptation in adult rats versus weanling rats or jejuno-ileal resection versus ICR with partial colon resection. Alternately, the higher dietary fat content in this study might have maximized the capacity for small bowel adaptation but the different fatty acid composition, n-3 LCPUFA versus n-6 PUFA, still impacted the large bowel adaptation.

The colon plays an important role in the absorption of fluid, electrolytes, and medium-chain triglycerides, produces shortchain fatty acids for energy salvage, hosts intestinal microbiota, and synthesizes vitamin K (23). Colonic adaptation is crucial in SBS and has previously been studied in adult rats (24-26) but not in infants. To the authors' knowledge, this is the first report on the effects of dietary fats on colonic adaptation in an infant SBS model. It is suspected that the increased colonic mucosal adaptation in the FO diet-fed ICR rats was associated with enhancing colonic function, including increased absorption of fluid and fat, and the production of PYY, responsible for the colonic brake phenomenon (21), leading to less diarrhea and steatorrhea. In addition, the antiinflammatory properties of n-3 EPA and DHA from FO are speculated to be of benefit to the increased susceptibility to inflammation and infection in ICR rats secondary to bacterial overgrowth in the residual small intestine (27). More detailed 
mechanisms identifying the beneficial effects of dietary FO on colonic adaptation need to be explored.

In conclusion, the high fat diet enriched with n-3 LCPUFA, EPA, and DHA, from FO increased colonic mucosal adaptation, and decreased diarrhea and fecal fat excretion in 20-d-old weanling rats after massive ICR, compared with the diet high in n-6 PUFA from CO. This study raises important questions regarding the dietary management of infants with SBS. Overall, research evidence from this and previous studies shows that high fat diets and diets high in LCPUFA are most beneficial in cases of SBS (4,5,7-11) and that low-fat diets impair postresection intestinal adaptation (6), despite that these findings are paradoxical to current wide-spread clinical practice. Infants with SBS are often given elemental formula containing less fat than human breast milk, having considerably high medium-chain fatty acids, and being dominated by n-6 PUFA. Therefore, additional studies especially clinical trials are needed to improve the enteral nutritional support to infants with SBS.

Acknowledgments. We thank Dr. April Ronca for providing the weanling rats, Ms. Hermina Borgerink for preparation of the histologic slides, and Ms. Beth Phifer for morphometric analyses.

\section{REFERENCES}

1. Feldman EJ, Dowling RH, McNaughton J, Peters TJ 1976 Effects of oral versus intravenous nutrition on intestinal adaptation after small bowel resection in the dog. Gastroenterology 70:712-719

2. Warner BW, Vanderhoof JA, Reyes JD 2000 What's new in the management of short gut syndrome in children? J Am Coll Surg 190:725-736

3. Morin CL, Grey VL, Garofalo C 1981 Influence of lipids on intestinal adaptation after resection. In: Robinson JW, Dowling RH, Riecken EO (eds) Mechanism in Intestinal Adaptation. MTP Press, Lancaster, United Kingdom, pp 175-185

4. Kollman KA, Lien EL, Vanderhoof JA 1999 Dietary lipids influence intestinal adaptation after massive bowel resection. J Pediatr Gastroenterol Nutr 28:41-45

5. Sukhotnik I, Mor-Vaknin N, Drongowski RA, Miselevich I, Coran AG, Harmon CM 2004 Effect of dietary fat on early morphological intestinal adaptation in a rat with short bowel syndrome. Pediatr Surg Int 20:419-424
6. Sukhotnik I, Shiloni E, Krausz M, Yakirevich E, Sabo E, Mogilner J, Coran AG, Harmon CM 2003 Low-fat diet impairs postresection intestinal adaptation in a rat model of short bowel syndrome. J Pediatr Surg 38:1182-1187

7. Sukhotnik I, Gork AS, Chen M, Drongowski RA, Coran AG, Harmon CM 2003 Effect of a high fat diet on lipid absorption and fatty acid transport in a rat model of short bowel syndrome. Pediatr Surg Int 19:385-390

8. Sukhotnik I, Mor-Vaknin N, Drongowski RA, Coran AG, Harmon CM 2004 Effect of dietary fat on fat absorption and concomitant plasma and tissue fat composition in a rat model of short bowel syndrome. Pediatr Surg Int 20:185-191

9. Vanderhoof JA, Garandjean CJ, Kaufman SS, Burkley KT, Antonson DL 1984 Effect of high percentage medium-chain triglyceride diet on mucosal adaptation following massive bowel resection in rats. JPEN J Parenter Enteral Nutr 8:685-689

10. Isaacs PE, Ladas S, Forgacs IC, Dowling RH, Ellam SV, Adrian TE, Bloom SR 1987 Comparison of the effects of ingested medium- and long-chain triglyceride on gall bladder volume and the release of cholecystokinin and other gut peptides. Dig Dis Sci 32:481-486

11. Vanderhoof JA, Park JH, Herrington MK, Adrian TE 1994 Effects of dietary menhaden oil on mucosal adaptation after small bowel resection in rats. Gastroenterology 106:94-99

12. Yang Q, Kock ND 2010 Intestinal adaptation following massive ileocecal resection in 20-day-old weanling rats. J Pediatr Gastroenterol Nutr 50:16-21

13. Vanderhoof JA 1996 Short bowel syndrome. Clin Perinatol 23:377-386

14. Goulet O, Ruemmele F 2006 Causes and management of intestinal failure in children. Gastroenterology 130:S16-S28

15. Bligh EG, Dyer WJ 1959 A rapid method of total lipid extraction and purification. Can J Biochem Physiol 37:911-917

16. Metcalfe LD, Schmitz AA, Peika JR 1966 Rapid preparation of fatty acid esters from lipids for gas chromatographic analysis. Anal Chem 38:514-515

17. Dahlqvist A 1984 Assay of intestinal disaccharidases. Scand J Clin Lab Invest 44:169-172

18. Park JH, Grandjean CJ, Hart MH, Baylor JM, Vanderhoof JA 1989 Effects of dietary linoleic acid on mucosal adaptation after small bowel resection. Digestion 44:57-65

19. Sangild PT 2006 Gut response to enteral nutrition in preterm infants and animals Exp Biol Med 231:1695-1711

20. DiBaise JK, Young RJ, Vanderhoof JA 2004 Intestinal rehabilitation and the short bowel syndrome: part 1. Am J Gastroenterol 99:1386-1395

21. Whang EE, Hines OJ, Reeve JR, Grandt D, Moser JA, Bilchik AJ, Zinner MJ, McFadden DW, Ashley SW 1997 Antisecretory mechanism of peptide YY in rat distal colon. Dig Dis Sci 42:1121-1127

22. Adrian TE, Savage AP, Fuessl HS, Wolfe K, Besterman HS, Bloom SR 1987 Release of peptide YY (PYY) after resection of small bowel, colon or pancreas in man. Surgery 101:715-719

23. Goulet O, Colomb-Jung V, Joly F 2009 Role of the colon in short bowel syndrome and intestinal transplantation. J Pediatr Gastroenterol Nutr 48:S66-S71

24. Kripke SA, De Paula JA, Berman JM, Fox AD, Rombeau JL, Settle RG 1991 Experimental short-bowel syndrome: effect of an elemental diet supplemented with short-chain triglycerides. Am J Clin Nutr 53:954-962

25. Gillingham MB, Dahly EM, Carey HV, Clark MD, Kritsch KR, Ney DM 2000 Differential jejunal and colonic adaptation due to resection and IGF-I in parenterally fed rats. Am J Physiol Gastrointest Liver Physiol 278:G700-G709

26. Xu J, Zhong Y, Jin D, Zhang H, Wu Z 2008 Effect of dietary fiber and growth hormone on colonic adaptation in short bowel syndrome treated by enteral nutrition. World J Surg 32:1832-1839

27. Vanderhoof JA 1995 Short bowel syndrome in children. Curr Opin Pediatr 7:560 568 